\title{
3. Die Konstante
}

des Stefan-Boltzmannschen Strahlungsgesetzes; neue absolute Messungen zwischen 20 und $450^{\circ} \mathrm{C}$; von Walther Gerlach.

\section{A. Versuchsanordnung.}

1. Die im folgenden mitgeteilten Resultate bilden eine Erweiterung der früher ausgeführten absoluten Messungen der Strahlungskonstanten $\sigma$ im Stefan-Boltzmannschen Gesetz

$$
S=\sigma T^{4} \text {. }
$$

Es wurde nach der gleichen Methode ${ }^{1}$ ), jedoch mit völlig neuer Anordnung gearbeitet. El weitert sind die Messungen insofern, als zu höheren Temperaturen übergegangen wurde. Jedoch beschränkte ich mich auf den Temperaturbereich zwischen 20 und $450^{\circ} \mathrm{C}$., da höhere Temperaturen weder mit hoher Genauigkeit festgelegt, noch mit relativ einfachen, noch leicht übersehbaren Anordnungen für Strahlungsmessungen reproduzierbar sind. Es wurde ferner stets bei demselben, ziemlich kleinen, Abstand von etwa $33 \mathrm{~cm}$ zwischen Thermosäule und schwarzem Körper gemessen, um niedere und hohe Temperaturerhöhungen des Empfängers zu erhalten, weil vor allem von Valentiner ${ }^{2}$ ) auf eine mögliche Fehlerquelle in meiner Anordnung hingewiesen wurde, die durch die Temperaturerhöhung des Meßstreifens bedingt sein soll.

2. Bezüglich Konstruktion und Verwendung dér Thermosäule sei auf frühere Angaben verwiesen; für die letzten Messungen (vgl. Anordnung III) stand mir ein vèrbessertes Paschengalvanorreter ${ }^{3}$ ) zur Verfügung, das bei höherer Instrumentempfindlichkeit vorzügliche Konstanz der Empfindlichkeit und der Ruhelage hatte. Diese Versuche haben demgemäß größere innere Genauigkeit. Es wurde ferner fast stets bei vollkommener austemperierter Meßanordnung ge-

1) W. Gerlach, Ann. d Phys. 88. p. 1. 1912.

2) S. Valentiner, Ann. d. Phys. 39. p. 489. 1912.

3) F. Paschen, Phys. Zeitschr. 14. p. 521. 1913. 
arbeitet, so daß die ,Gangbeobachtungen“" 1) nur selten erforderlich waren. Der Streifen ist mit Platinmohr nach Lummer-Kurlbaums elektrolytischem Verfahren dick platiniert, er hat ein schönes, gleichmäßig samtschwarzes Aussehen.

3. Fig. 1 gibt eine Skizze der Versuchsanordnung. $S$ ist der schwarze Körper, der unten näher beschrieben ist. Er steht hinter einem großen Wassertrog $W$ mit Ausschnitt zum

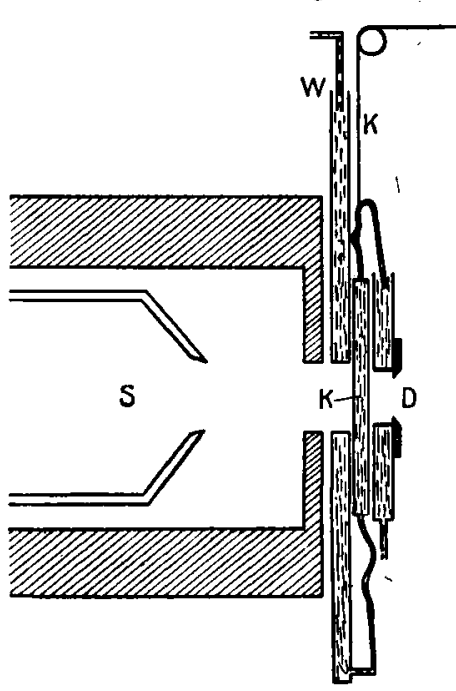

Fig. 1. Durchgang der Strahlung; an ihm liegt eine ebenfalls von Wasser durchflossene Zugklappe $K$ an; vor dieser steht, gleichfalls von Wasser gekühlt, das Hauptstrahlungsdiaphragma $D$, leicht auswechselbar in einen großen Metallschirm eingesetzt. Es war in eine $5 \mathrm{~mm}$ dicke Kupferplatte konisch eingedreht und dann abgeschliffen. Die Dimensionen der beiden benutzten Diaphragmen wurden durch wiederholte Messungen auf einem Zeißkomparator ermittelt; sie betrugen $D_{1}=1,6513 \mathrm{~cm}^{2}$, $D_{2}=0,794_{9} \mathrm{~cm}^{2}$. Durch Benutzung derselben Wassermenge zur Kühlung von Blenden, Diaphragma und Klappen war deren l'emperatur gleich und lange Zeit auf weniger als $0,1^{\circ}$ konstant. - Im Abstand $R$ vom Hauptdiaphragma stand die absolute Thermosäule, durch Blenden vor Luftströmungen und diffuser Strahlung geschützt. Verschiedentlich wurden in den Strahlengang Blenden mit enger Öffnung eingeschaltet, um die Anordnung auf Abwesenheit diffuser und reflektierter Strahlung zu prüfen. Die Temperatur der Klappe wurdo durch ein Thermometer (in zehntel Grad geteilt) gemessen, das in metallischem Kontakt mit derselben war. Die Entfernung $R$ betrug bei

Anordnung I $331,12 \mathrm{~mm}$

$$
\text { II } 331,12, " \quad \text { (anderes Thermosäulengehäuse); }
$$

1) W. Gerlach, 1. c. p. 20. 
sie war durch ein sorgfältig ausgewertetes Stichmaß gegeben, das zwischen Diaphragma und Thermosäulengehäuse horizontal eingeschaltet war. Die Dicke des letzteren bis zu dem die Empfängerfläche definierenden Silberspalt wurde mit einem Sphärometer gemessen.

4. Fig. 2 gibt die Konstruktion des elektrisch geheizten schwarzen Körpers. Der innere Hohlraum $S$ war aus $6 \mathrm{~mm}$ dickem Kupfer, alle Teile hart verlötet, geschwärzt. Er war

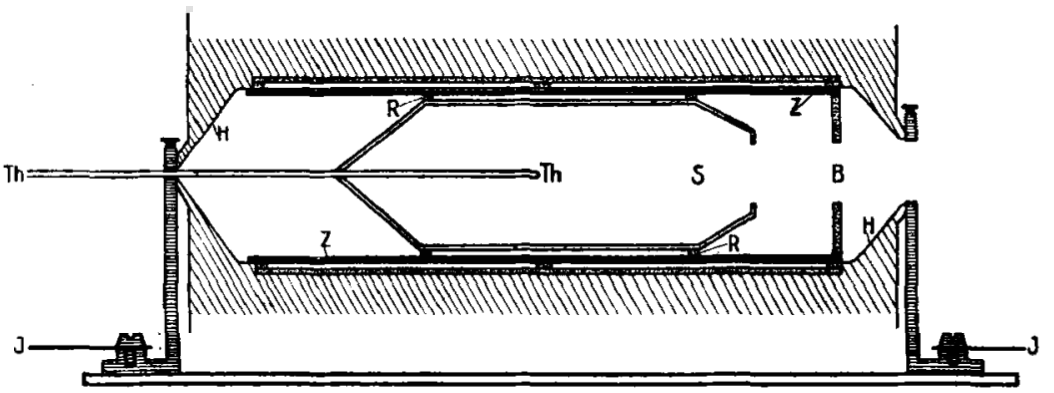

Messing Waint Asbest

Fig. 2. Schwarzer Körper.

mit zwei Ringen $R$ aus Asbestschnur in einem etwas weiteren, dünnen Tonzylinder $\boldsymbol{Z}$ gehalten, also von einem Luftraum umgeben. Um den Tonzylinder war das Heizblech $H$ aus $0,002 \mathrm{~mm}$ dickem Nickelblech gelegt, gehalten mit einigen Asbestschnüren; durch deren Dicke war ein zweiter Luftmantel gegèben - einige Millimeter -, begrenzt durch einen Asbestzylinder, den dann weiter die - durch Schraffierung angedeutete - dicke Wärmeisolierung aus Asbestwolle und Kieselguhr umgab. Die Stromzuführung erfolgte vorne und hinten, wo das Heizblech zusammengebogen und mittels Ring und Rundklemme an metallische Stützen angeschlossen war. $A$ sind Asbestringe, Th das die Temperatur des Strahlers messende Thermoelement. Die Wärmeisolation war gut, die Temperaturkonstanz für die Dauer von mehreren Strahlungsmessungen gleichfalls völlig ausreichend. Die Temperaturverteilung im Hohlraum und an der Wand, sowie im hinteren Luftraum war völlig gleichmäßig, wie Messungen mit Thermoelementen und Platinwiderstandsthermometer ergaben. 


\section{B. Temperaturmessung des Strahlers.}

5. Die Temperaturmessung erfolgte mittels eines Thermoelements aus $0,2 \mathrm{~mm}$ dickem Eisen- und Konstantandraht (von Hartmann \& Braun). Die Drahtstücke waren nach den gebräuchlichen Methoden auf innere Homogenität unter-

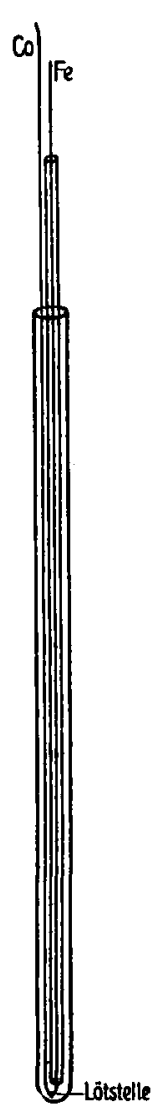

Fig. 3.

Thermoelement. sucht; sie waren nach intensivem Ausglïhen und stundenlanger Erhitzung im Heizkasten auf zirka $200^{\circ} \mathrm{C}$. fast absolut gleichförmig. Durch eine Spur Silber stumpf aneinander gelötet, waren sie (Fig. 3) in Porzellanröhrchen isoliert, deren äußeres an der Lötstelle des Thermoelements sehr dünnwandig zugeblasen war. Die ,kalten" Lötstellen waren in oinem Petroleumbad auf $0^{\circ} \mathrm{C}$. gehalten.

6. Die Eichung der beiden benutzten Elemente wurde nach den in der Reichsanstalt und dem Bureau of Standards ausgearbeiteten Methorlen durchgeführt. Es wurden benutzt:

Schmelzendes Eis . . . . . $0,0^{\circ}$

Siedendes Wasser . . . . . 100,0

Siedendes Naphthalin . . . . 218,0

Siedender Schwefel . . . . . 444,6

Eine Umrechnung mit den neuen, von der Reichsanstalt ${ }^{1}$ ) angegebenen Werten $217,96^{\circ}$ und $444,55^{\circ}$ wurde unterlassen, da diese Differenz auf die Konstante des Strahlungsgesetzes ohne Einfluß ist.

Zum Sieden aller Substanzen diente derselbe Apparat, der elektrisch geheizt wurde. Die Höhe der Heizspule betrug $10 \mathrm{~cm}$, um mindestens $5 \mathrm{~cm}$ überragt von der siedenden Flüssigkeit, zur Vermeidung etwaiger Überhitzung. Es erwies sich als besonders günstig, zwischen der Heizspule, die auf einem dünnen Tonzylinder aufgewickelt war, und Glassiederohr eine lockere Schicht von Asbestschnur bis an das Ende der siedenden Flüssigkeit zu bringen; das Sieden erfolgte dann merklich gleichmäBiger als mit Luftzwischenraum oder mit fester Isolierpackung. Bei geringster Energiezufuhr (41/2 Amp. bei 60 Volt) siedete der Schwefel viele Stunden lang absolut gleichmäßig.

1) Verh. d. D. Phys. Ges. 18. p. 1. 1916. 
Tabelle I gibt die Eichungsmessungen des Thermoelements. Die Chemikalien waren von Kahlbaum bezogen.

Tabelle I.

\begin{tabular}{|c|c|c|c|c|}
\hline $\begin{array}{c}\text { Messungen } \\
\text { mm }\end{array}$ & Substanz & $\begin{array}{l}\text { Angenommene } \\
\text { Siedetemperatur } \\
\text { bei } 760 \mathrm{~min}\end{array}$ & $\begin{array}{c}\text { Beob. } \\
\text { EMK } \times 10^{2}\end{array}$ & $\begin{array}{c}\text { Ab- } \\
\text { weichungen } \\
\text { vom Mittel }\end{array}$ \\
\hline$\frac{\text { Nov. }}{\text { Dez. }} 1913$ & Wasser & $100,0^{\circ}$ & 0,5040 & $0,05 \%$ \\
\hline $\begin{array}{l}\text { Nov. } 1913 \\
\text { Dez. } 1913 \\
\text { Juni } 1914 \\
\text { Okt. } 1913 \\
\text { Dez. } 1913 \\
\text { Juni } 1914\end{array}$ & Schwefel & $444,6^{\circ}$ & $\begin{array}{l}1,1382 \\
1,1375 \\
1,1381 \\
2,3630 \\
2,3633 \\
2,3630\end{array}$ & $\begin{array}{r}+0,03 \% \\
-0,04 \% \\
+0,02 \% \\
<0,01 \%\end{array}$ \\
\hline
\end{tabular}

7. Zur Kontrolle standen mir einige Quecksilbertherm.ometer mit Prüfungsscheinen und ein gleichfalls von der Reichsanstalt geprüftes Platinwiderstandsthermometer zur Verfügung. Die Eichungen der Thermometer, die in dem Siedeapparat wiederholt wurden, lieferten mit den Prüfungstabellen übereinstimmende Resultate. Die Genauigkeit meines Platinthermometers ist bei $450^{\circ}$ nur $z u \pm 1^{\circ}$ angegeben. $D a$ bei einigen Strahlungsmessungen die Temperatur außer mit dem Thermoelement auch mit dem Widerstandsthermometer gemessen wurde, sei der Vergleich der beiden Temperaturskalen angegeben:

Tabelle II.

\begin{tabular}{|c|c|c|}
\hline $\begin{array}{l}\text { Thermoelementtemp. } \\
\text { nach meiner Eichung }\end{array}$ & $\begin{array}{l}\text { Platintemperatur nach } \\
\text { der Tabelle der P. T. R. }\end{array}$ & Differenz $\mathrm{Pt}-\mathrm{Th}$ \\
\hline $\begin{array}{l}\left.101,3^{1}\right) \\
\left.143,3^{1}\right) \\
\left.214,3^{1}\right) \\
\left.217,5^{2}\right) \\
\left.248,6^{1}\right) \\
\left.311,8^{1}\right) \\
\left.373,0^{1}\right) \\
\left.430,3^{1}\right) \\
\left.443,3^{2}\right)\end{array}$ & $\begin{array}{l}\left.101,0^{1}\right) \\
\left.142,9^{1}\right) \\
\left.214,5^{1}\right) \\
\left.218,0^{2}\right) \\
\left.249,0^{1}\right) \\
\left.312,7^{1}\right) \\
\left.374,4^{1}\right\} \\
\left.430,7^{1}\right) \\
\left.443,8^{2}\right)\end{array}$ & $\begin{array}{l}-0,3^{0}=-0,30 \% \\
-0,4^{0}=-0,28 \% \\
+0,2^{\circ}=+0,09 \% \\
+0,5^{0}=+0,23 \% \\
+0,4^{0}=+0,16 \% \\
+0,9^{0}=+0,29 \% \\
+1,4^{0}=+0,38 \% \\
+0,4^{0}=+0,09 \% \\
+0,5^{0}=+0,12 \%\end{array}$ \\
\hline
\end{tabular}

1) Messungen in einem Vergleichsofen.

2) Siedepunktsmessungen von Naphthalin und Schwefel (beobachtete Werte ohne Barometerkorrektion). 


\section{Die Strahlungamessungen.}

8. Zunächst wurde die Thermosäule auf vollkommene Isolation zwischen MeBstreifen und Thermoelementen geprüft. Ferner wurde untersucht, ob die Einstellung des Galvanometers bei Strahlungs- und Stromheizungen des Streifens sich gleichartig vollzog ${ }^{1}$ ), und drittens wurde die Proportionalität zwischen den Angaben der Thermosäule (Galvanometerausschläge) mit der Stromheizung geprüft. Alle Bedingungen waren vollkommen erfüllt, für die Proportionalitätsmessungen sei eine beliebige Meßreihe aus einem Versuch gegeben (Tabelle III).

Tabelle III.

\begin{tabular}{c|c|c|c}
\hline \hline Heizstrom & Ausschlag & Proportionalität & $\begin{array}{c}\text { Differenz gegen } \\
\text { Mittel in Proz. }\end{array}$ \\
\hline 0,01313 & 71,4 & 2414 & 0,5 \\
0,02699 & 303,6 & 2400 & 0,08 \\
0,03370 & 473,8 & 2397 & 0,2 \\
0,03865 & 621,6 & 2403 & 0,04 \\
0,04330 & 782,8 & 2395 & 0,3
\end{tabular}

Die Messung des Stromes erfolgte durch Kompensation an einem Normalohm, die Spannung an den Streifenenden zur Berechnung der elektrischen Energie wurde gleichfalls durch Kompensation gemessen. Spannungsmessungen durch Potentialdrähte, welche in einiger Entfernung von den Streifenenden angelötet waren, ergaben gleiches Resultat.2)

9. Vor Beginn der Strahlungsmessungen wurde die Temperaturverteilung im Hohlraum untersucht. Erst nachdem diese konstant geworden war (was sehr bald geschah), wurde mit den Messungen begonnen. Die Heizung des schwarzen Körpers erfolgte derart, daß mit hoher Stromstärke (100 Amp. bei 4 Volt) angeheizt und dann durch Verminderung oder Ausschalten des Stromes, je nach der Temperatur, Konstanz der Temperatur eingestellt wurde. Öffnen und Schließen der Klappe erfolgte vom Beobachtungsfernrohr aus, Nullpunkt des Galvanometers, Nulleinstellung der Thermosäule und Einstellung des Ausschlags war bei fast allen Messungen konstant.

1) W. Gerlach, l. c. und Ann. d. Phys. 41. p. 99.1913.

2) W. Gerlach, Physik. Zeitschr. 17. p. 150. 1916. 
10. Tabellen IV, V und VI geben die - im November, Dezember 1913 und Januar 1914 ausgeführten - Messungen, nach Temperaturen geordnet. Die Messungen der Tabelle V sind nach Umbau und never Justierung der Anordnung 1 ausgeführt, die Messungen der Tabelle VI mit dem kleineren I)iapbragma ( $D$ 2) mit gleichfalls vollkommen nou aufgestellter Anordnung. Die Berechnung der Versuchswerte erfolgte nach der früher abgeleiteten Formel

$$
A i^{2} \omega=\frac{\sigma}{\pi}\left(T_{1}^{4}-T_{2}^{4}\right) \frac{F_{1} \cdot F_{3}}{R^{2}} \cdot f\left(F_{1}, F_{2}, R\right),
$$

worin $f\left(F_{1}, F_{2}, R\right)$ die Korrektion wegen der nicht gleichen Abstände aller Flächenelemente des Strahlers von denen des Empfängers bedeutet. $T_{2}$ ist die Temperatur des bei geschlossener Klappe zur 'Thermosäule strahlenden Hohlraumes.

Tabelle IVa.

Anordnung Nr. 1.

\begin{tabular}{|c|c|c|c|c|c|c|}
\hline$T_{1}{ }^{\circ} \mathrm{C}$ & $T_{2}{ }^{\circ} \mathrm{C}$ & $\begin{array}{c}\sigma \times 10^{13} \\
\text { beob. }\end{array}$ & $\begin{array}{c}\text { Reflex. } \\
\text { Korr. }\end{array}$ & $\underset{\text { Korr. }}{\mathrm{CO}_{2}}$ & $\begin{array}{l}\mathrm{H}_{8} \mathrm{O} \\
\text { Korr. }\end{array}$ & $\sigma \times 10^{12}$ \\
\hline $\begin{array}{l}158,8 \\
158,8 \\
158,8 \\
159,4 \\
232,1 \\
232,1 \\
238,4 \\
238,4 \\
238,4 \\
239,7\end{array}$ & $\begin{array}{l}20,60 \\
20,60 \\
20,60 \\
20,60 \\
20,05 \\
20,05 \\
20.05 \\
20,05 \\
20,05 \\
20,05\end{array}$ & $\begin{array}{l}\mathbf{5 , 7 5} \\
\mathbf{5 , 7 1} \\
\mathbf{5 , 7 1} \\
\mathbf{5 , 6 4} \\
\mathbf{5 , 6 6} \\
\mathbf{5 , 6 6} \\
\mathbf{5 , 8 0} \\
\mathbf{5 , 8 0} \\
\mathbf{5 , 6 9} \\
\mathbf{5 , 6 5}\end{array}$ & $\stackrel{i}{0}$ & $0,4 \%$ & $0,2 \%$ & $\begin{array}{l}\mathbf{5 , 8 5} \\
\mathbf{5 , 8 1} \\
\mathbf{5 , 8 1} \\
\mathbf{5 , 7 4} \\
\mathbf{5 , 8 0} \\
\mathbf{5 , 8 0} \\
\mathbf{5 , 9 5} \\
\mathbf{5 , 9 5} \\
\mathbf{5 , 8 3} \\
\mathbf{5 , 7 9}\end{array}$ \\
\hline $\begin{array}{l}292,1 \\
311,9 \\
312,7 \\
313,6 \\
314,3 \\
317,6\end{array}$ & $\begin{array}{l}21,00 \\
22,30 \\
22,30 \\
22,30 \\
22,30 \\
22,30\end{array}$ & $\begin{array}{l}\mathbf{5 , 7 3} \\
\mathbf{5 , 6 9} \\
\mathbf{5 , 7 6} \\
\mathbf{5 , 5 7} \\
\mathbf{5 , 7 3} \\
\mathbf{5 , 6 4}\end{array}$ & $\begin{array}{l}0 \\
0 \\
+ \\
0 \\
0 \\
0\end{array}$ & $1,2 \%$ & $0 \%$ & $\begin{array}{l}\mathbf{5 , 9 0} \\
\mathbf{5 , 8 6} \\
\mathbf{5 , 9 3} \\
\mathbf{5 , 7 3} \\
\mathbf{5 , 9 0} \\
\mathbf{5 , 8 1}\end{array}$ \\
\hline $\begin{array}{l}342,6 \\
343,1 \\
346,6 \\
351,1 \\
352,0 \\
355,5 \\
356,5 \\
359,7\end{array}$ & $\begin{array}{l}22,30 \\
22,30 \\
22,30 \\
22,30 \\
22,30 \\
22,30 \\
22,30 \\
22,30\end{array}$ & $\begin{array}{l}\mathbf{5 , 6 0} \\
\mathbf{5 , 6 0} \\
\mathbf{5 , 7 4} \\
\mathbf{5 , 5 3} \\
\mathbf{5 , 7 4} \\
\mathbf{5 , 6 1} \\
\mathbf{5 , 6 4} \\
\mathbf{5 , 6 7}\end{array}$ & 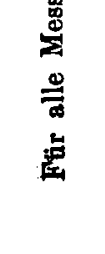 & $1,0 \%$ & $0 \%$ & $\begin{array}{l}\mathbf{5 , 7 6} \\
\mathbf{5 , 7 6} \\
\mathbf{5 , 9 0} \\
\mathbf{5 , 6 9} \\
\mathbf{5 , 9 0} \\
\mathbf{5 , 7 7} \\
\mathbf{5 , 8 0} \\
\mathbf{5 , 8 3}\end{array}$ \\
\hline $\begin{array}{l}429,5 \\
433,5 \\
435,3 \\
436,2\end{array}$ & $\begin{array}{l}21,00 \\
21,00 \\
21,00 \\
21,00\end{array}$ & $\begin{array}{l}\mathbf{5 , 6 0} \\
\mathbf{5 , 7 1} \\
\mathbf{5 , 6 4} \\
\mathbf{5 , 5 5}\end{array}$ & & $0,7 \%$ & $0 \%$ & $\begin{array}{l}\mathbf{5 , 7 4} \\
\mathbf{5 , 8 5} \\
\mathbf{5 , 7 8} \\
\mathbf{5 , 6 9}\end{array}$ \\
\hline
\end{tabular}


Tabelle IVb.

Mittelwerte der Messungen mit Anordnung Nr. 1.

\begin{tabular}{|c|c|c|c|c|c|c|}
\hline \multicolumn{2}{|c|}{$\begin{array}{c}\text { Temperatur- } \\
\text { bereich }\end{array}$} & $10^{12}$ beob & \multicolumn{4}{|c|}{$\sigma \times 10^{12}$ Korr. } \\
\hline \multicolumn{2}{|c|}{$\begin{array}{c}\sim 160 \\
230-240 \\
\sim 290 \\
310-320 \\
340-360 \\
430-440\end{array}$} & $\begin{array}{l}\mathbf{5 , 7 0} \\
\mathbf{5 , 6 9} \\
\mathbf{5 , 7 3} \\
\mathbf{5 , 6 8} \\
\mathbf{5 , 6 4} \\
\mathbf{5 , 6 3}\end{array}$ & & $\begin{array}{l}, 80 \\
, 83 \\
, 90 \\
, 85 \\
, 80 \\
, 77 \\
\end{array}$ & & \\
\hline \multicolumn{7}{|c|}{$\begin{array}{c}\text { Tabelle Va. } \\
\text { Anordnung Nr. } 2 .\end{array}$} \\
\hline$T_{1}{ }^{\circ} \mathrm{C}$ & $T_{8}{ }^{\circ} \mathrm{C}$ & $\begin{array}{c}\sigma \times 10^{12} \\
\text { beob. }\end{array}$ & $\begin{array}{c}\text { Reflex. } \\
\text { Korr. }\end{array}$ & $\begin{array}{c}\mathrm{CO}_{2} \\
\text { Korr. }\end{array}$ & $\begin{array}{l}\mathrm{H}_{2} \mathrm{O} \\
\mathrm{K} \text { orr. }\end{array}$ & $\sigma \times 10^{12}$ \\
\hline $\begin{array}{l}100,6 \\
101 ; 5 \\
102,6 \\
103,5\end{array}$ & $\begin{array}{l}18,40 \\
18,40 \\
18,40 \\
18,40\end{array}$ & $\begin{array}{l}\mathbf{5 , 6 9} \\
\mathbf{5 , 7 0} \\
\mathbf{5 , 7 2} \\
\mathbf{5 , 7 1}\end{array}$ & $\begin{array}{l}0=1 \\
1 \\
0 \\
x \\
0 \\
0\end{array}$ & $0 \%$ & $0 \%$ & $\begin{array}{l}\mathbf{5 , 7 9} \\
\mathbf{5 , 8 0} \\
\mathbf{5 , 8 2} \\
\mathbf{5 , 8 1}\end{array}$ \\
\hline $\begin{array}{l}193,7 \\
202,1 \\
204,7\end{array}$ & $\begin{array}{l}20,00 \\
19,90 \\
19,90\end{array}$ & $\begin{array}{l}\mathbf{5}, 66 \\
\mathbf{5}, 73 \\
\mathbf{5}, 70\end{array}$ & $\begin{array}{l}= \\
+ \\
+\end{array}$ & $0,2 \%$ & $0,2 \%$ & $\begin{array}{l}\mathbf{5 , 7 8} \\
5,85 \\
5,82\end{array}$ \\
\hline
\end{tabular}

Tabelle Vb.

Mittelwerte der Messungen mit Anordnung Nr. 2.

\begin{tabular}{c|c|c}
\hline \hline $\begin{array}{c}\text { Temperatur- } \\
\text { bereich }\end{array}$ & $\sigma \times 10^{12}$ beob. & $\sigma \times 10^{12}$ Korr. \\
\hline \hline$\sim 100$ & 5,70 & 5,83 \\
$\sim 200$ & 5,70 & $\frac{5,82}{}$ \\
& & Mittel $: 5,83 \cdot 10^{-12}$ Watt cm $^{-2} \mathrm{Grad}^{-4}$
\end{tabular}

11. Das Reflektionsvermögen des Empfängers wurde nicht besonders bestimmt, es ist hier wie früher eine Korrektion von 0,10 Watt $\mathrm{cm}^{-2} \mathrm{grad}^{-4}$ angebracht, welche wohl bis auf einige wenige Promille als richtige Korrektionsgröße angesehen werden darf. ${ }^{1}$ ) Die Versuche mit Anordnung 1 und 2 ergeben einen etwas kleineren Wert als Anordnung 3. Das bei den erstgenannten Versuchen verwandte Diaphragma war so gro 3

1) Z. B. W. W. Coblentz, Bull. Bur. Stand. 9. p. 283.1913. 
Tabelle VIa.

Anordnung Nr. 3.

\begin{tabular}{|c|c|c|c|c|c|c|}
\hline$T_{1}{ }^{\circ} \mathrm{C}$ & $T_{2}{ }^{\circ} \mathrm{C}$ & $\begin{array}{c}\sigma \times 10^{12} \\
\text { beob. }\end{array}$ & $\begin{array}{c}\text { Reflex. } \\
\text { Korr. }\end{array}$ & $\begin{array}{c}\mathrm{CO}_{2} \\
\text { Korr. }\end{array}$ & $\begin{array}{c}\mathrm{H}_{2} \mathrm{O} \\
\text { Korr. }\end{array}$ & $\sigma \times 10^{12}$ \\
\hline $\begin{array}{l}254,9 \\
257,1 \\
257,1 \\
258,7 \\
259,7\end{array}$ & $\begin{array}{l}15,60 \\
15,50 \\
15,50 \\
15,60 \\
15,60\end{array}$ & $\begin{array}{l}5,76 \\
5,78 \\
5,78 \\
5,78 \\
5,79\end{array}$ & \multirow{4}{*}{ 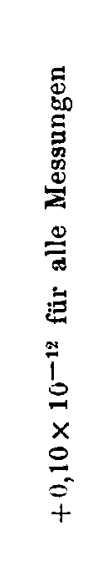 } & \multirow{2}{*}{$\begin{array}{l}0,4 \% \\
1,2 \%\end{array}$} & $0 \%$ & $\begin{array}{l}\mathbf{5 , 8 8} \\
\mathbf{5 , 9 0} \\
\mathbf{5 , 9 0} \\
\mathbf{5 , 9 0} \\
\mathbf{5 , 9 1}\end{array}$ \\
\hline $\begin{array}{l}303,8 \\
303,8 \\
314,4 \\
315,1\end{array}$ & $\begin{array}{l}15,50 \\
15,50 \\
16,10 \\
16,10\end{array}$ & $\begin{array}{l}\mathbf{5 , 7 0} \\
\mathbf{5 , 7 0} \\
\mathbf{5 , 7 9} \\
\mathbf{5 , 8 0}\end{array}$ & & & $0 \%$ & $\begin{array}{l}\mathbf{5 , 8 7} \\
\mathbf{5 , 8 7} \\
\mathbf{5 , 9 6} \\
\mathbf{5 , 9 7}\end{array}$ \\
\hline $\begin{array}{l}343,1 \\
343,1 \\
344,0 \\
358,9 \\
358,9\end{array}$ & $\begin{array}{l}15,00 \\
15,00 \\
15,00 \\
14,60 \\
14,60\end{array}$ & $\begin{array}{l}\mathbf{5 , 7 1} \\
\mathbf{5 , 7 1} \\
\mathbf{5 , 7 4} \\
\mathbf{5 , 7 7} \\
\mathbf{5 , 7 7}\end{array}$ & & $1,0 \%$ & $0 \%$ & $\begin{array}{l}\mathbf{5 , 8 7} \\
\mathbf{5 , 8 7} \\
\mathbf{5 , 9 0} \\
\mathbf{5 , 9 3} \\
\mathbf{5 , 9 3}\end{array}$ \\
\hline $\begin{array}{l}394,3 \\
394,3 \\
395,4 \\
400,5\end{array}$ & $\begin{array}{l}15,70 \\
15,70 \\
15,70 \\
15,70\end{array}$ & $\begin{array}{l}\mathbf{5 , 7 0} \\
\mathbf{5 , 7 0} \\
\mathbf{5 , 6 7} \\
\mathbf{5 , 6 9}\end{array}$ & & $0,7 \%$ & $0 \%$ & $\begin{array}{l}5,84 \\
5,84 \\
5,81 \\
5,83\end{array}$ \\
\hline
\end{tabular}

Tabelle VIb.

Mittelwerte der Messungen mit Anordnung Nr. 3.

\begin{tabular}{c|c|c}
\hline $\begin{array}{c}\text { Temperatur- } \\
\text { bereich }\end{array}$ & $\sigma \times 10^{12}$ beob. & $\sigma \times 10^{12}$ Korr. \\
\hline $250-260$ & 5,78 & 5,90 \\
$300-320$ & 5,75 & 5,92 \\
$340-360$ & 5,74 & 5,90 \\
$390-400$ & 5,69 & 5,85 \\
\hline
\end{tabular}

als möglich, um eben noch alle Strahlung aus dem Innern des schwarzen Körpers auszunutzen, aber keine falsche Strahlung z. B. von kälteren Teilen zuzulassen. Es ist nicht unmöglich, daß deshalb Anordnung 3 mit einem wesentlich kleineren Diaphragma in höherem MaBe schwarze Strahlung ergab als 1 und 2. Da jedoch sichere Grundlagen hierfür fehlen und die Abweichung noch innerhalb der Meßgenauigkeit liegt, sind die Gesamtmittel, Tabelle IVb, Vb, VIb, in gleicher Weise zur Berechnung des Endwertes herangezogen. Die Spalten mit „,CO $\mathrm{CO}_{2}$-Korr. und $\mathrm{H}_{2} \mathrm{O}$-Korr. geben die experimentell 
ermittelten Korrektionen für die Absorption der Strahlung in Kohlensäure- und Wasserdampfgehalt der Atmosphäre. ${ }^{1}$ )

\section{Resultat.}

12. Als Endwert folgt aus 52 voneinander unabhängigen Strahlungsmessungen, deren jeder mindestens 2 Strahlungs- und Strombeobachtungen zugrunde liegen, für die Konstante des Stefan-Boltzmannschen Strahlungsgesetses zwischen $300^{\circ}$ und $700^{\circ}$ absolut

$$
\underline{\sigma}=5,85 \times 10^{-12} \mathrm{Watt} \mathrm{cm}^{-2} \mathrm{Grad}^{-4} .
$$

Dieser Wert steht in guter Übereinstimmung zwischen mit dem früher zwischen $0^{\circ}$ und $100^{\circ} \mathrm{C}$ von mir bestimmten Werte $5,9 \cdot 10^{-12}$. Die Einzelwerte der neuen Bestimmungen schwanken zwischen 5,77 und 5,93, woraus eine Genauigkeit ron $1-1 \frac{1}{2}$ Proz. folgt.

13. Die exakte absolute Messung der Strahlungskonstanten gestattet eine Prüfung der Planckschen Strahlungstheorie. Planck berechnet mit Hilfe der Strahlungskonstanten $\sigma$ des Integralgesetzes und der Konstanten $c_{2}$ (resp. $b=\lambda_{m} T$ ) des spektralen Strahlungsgesetzes Werte für die universellen Konstanten $h$ und $k$, sowie für das elektrische Elementarquantum $e$ und die Lohschmidtsche Zahl $N$. Da aber der Wert ron $c_{2}$ in der dritten resp. vierten Potenz eingeht, so ist die Prüfung der Theorie wesentlich durch die Genauigkeit der Bestimmung dieses Strahlungswertes bedingt. Durch die Ausführung direkter experimenteller Messungen der Konstanten $h$ des Energiequantums ist aber die Möglichkeit gegeben, doppelte Beziehungen zu errechnen. Nach Millikans ${ }^{2}$ ) neuen photoelektrischen Messungen ist unter Annahme der GröBe des Elerentarquantums zu $4,77 \times 10^{-10}$ elst. Einh.

$$
h=6,58 \times 10^{-27} \mathrm{erg} / \mathrm{sec} .
$$

Aus Plancks Formel $h=\frac{a e_{z}{ }^{4}}{48 \pi} \frac{1}{\alpha}$ folgt mit $h=6,58$ und $a=4 \sigma / c=7,80 \times 10^{-15} \mathrm{erg} / \mathrm{cm}^{-3} \mathrm{Grad}^{-4}\left(\sigma=5,85 \times 10^{-12} \mathrm{Watt}\right.$ $\left.\mathrm{cm}^{-2} \mathrm{Grad}^{-4}\right)$ für die Konstante $c_{2}$ der Wert 1,4250.

1) Vgl. die vorstehende Arbeit.

2) R. A. Millikan, Phys. Rev. (2) 7. p. 18. 1916. 
Die neuesten Messungen der Reichsanstalt von Warburg und Müller ${ }^{1}$ ) lassen die Werte zu

$$
C_{2}=1,4250,1,4300,1,4400,
$$

und zwar folgt der niedrigste Wert aus Messungen, bei welchen die Temperatur nach dem Stefan-Boltzmannschen Gesetz erhalten war. Berechnet man mit diesem $c_{2}$-Wert nach Plancks Theorie das Elementarquantum der Elektrizität, so folgt für dieses

$$
e=4,83 \times 10^{-10} \text { elst. Einh., }
$$

während der beste experimentelle Wert nach Millikan ist:

$$
e=4,77 \times 10^{-10} \text { elst. Einh.; }
$$

zum Vergleich seien die $e$ - und $h$-Werte angegeben, die aus

\begin{tabular}{|c|c|c|c|c|}
\hline \multirow[t]{2}{*}{ Mit $c_{2}$} & \multicolumn{2}{|c|}{$\begin{array}{c}\text { folgt nech Plencke Theorie } \\
\qquad=5,55 \times 10^{-17}\end{array}$} & \multicolumn{2}{|c|}{$\begin{array}{l}\text { für das Elementarquantum au } \\
\sigma=5,85 \times 10^{-19}\end{array}$} \\
\hline & $6 \times 10^{10}$ & $h \times 10^{27}$ & $e \times 10^{10}$ & $h \times 10^{97}$ \\
\hline $\begin{array}{l}1,425 \\
1,430 \\
1,440\end{array}$ & $\begin{array}{l}4,58 \\
4,63 \\
4,73\end{array}$ & $\begin{array}{l}6,23 \\
6,31 \\
6,50\end{array}$ & $\begin{array}{l}4,83 \\
4,88 \\
4,98\end{array}$ & $\begin{array}{l}6,57 \\
6,66 \\
-\end{array}$ \\
\hline
\end{tabular}
Plancks Theorie für $\sigma=5,55$ (Westphal) und 5,85 (Gerlach) unter Zugrundelegung der $c_{2}$-Werte $1,425,1,430,1,440$ folgen:

Für die Lohschmidtsche Zahl folgt nach Plancks Theorie mit meinem $\sigma$-Wert $N=60,0 \times 10^{22} \quad\left(c_{2}=1,425\right)$ und $N=59,3\left(c_{2}=1,430\right)$. Direkt gemessen ist z. B. von Nordlund $59,1 \times 10^{22}$.

Tübingen, P'aysikal. Inst. der Universität, März 1916.²)

1) E. Warburg u. C. Müller, Ann. d. Phys. 48. p. 410. 1915.

2) Auszug aus der Tübinger Habilitationsschrift des Verfassers.

(Eingegangen 10. April 1916.) 